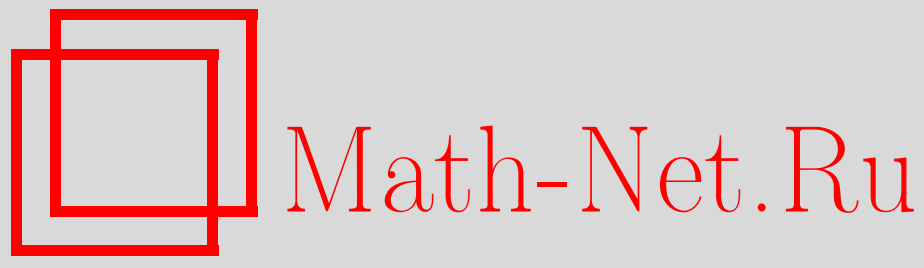

Д. А. Панов, Специальные точки поверхностей в трехмерном проективном пространстве, Функи. анализ и его прил., 2000, том 34, выпуск 4, 49-63

DOI: https://doi.org/10.4213/faa325

Использование Общероссийского математического портала MathNet.Ru подразумевает, что вы прочитали и согласны с пользовательским соглашением

http://www.mathnet.ru/rus/agreement

Параметры загрузки:

IP : 34.229 .45 .116

26 апреля 2023 г., 14:28:58

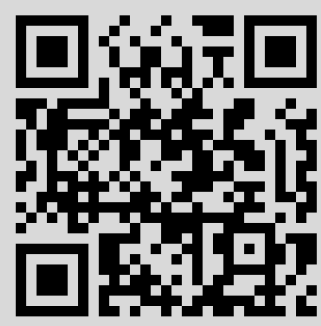


Функциональный анализ и его приложения

2000, т. 34, вып. 4, с. 49-63

УДК 517

\title{
Специальные точки поверхностей в трехмерном проективном пространстве*
}

\author{
(C) 2000. Д. А. ПАНОВ
}

\section{Введение}

Целью настоящей работы является исследование вещественной проективной топологии поверхностей в трехмерном пространстве. Мы построим комплекс типа васильевского [1], описывающий стратификацию точек поверхности.

Понятие $k$-струи поверхности, вложенной в трехмерное пространство, является обобщением понятия касательной плоскости.

ОПРЕДЕЛЕНИЕ 1. Две гладкие поверхности $M_{1}$ и $M_{2}$, расположенные в трехмерном пространстве, принадлежат одной $k$-струе в точке $x \in M_{1} \cap M_{2}$, если расстояние от точки $y \in M_{1}$ до поверхности $M_{2}$ представляет собой $o\left(|x-y|^{k}\right)$ при $y \rightarrow x$.

Поверхность, вложенная в аффинное пространство, допускает естественную стратификацию. А именно, можно показать (теорема 1), что 3-струя коориентированной поверхности, лежащей в $\mathbb{R}^{3}$, приводится аффинным преобразованием $\mathrm{K}$ одному из 22 видов. Таким образом, мы будем различать на поверхности 22 типа точек. Эта стратификация является детализацией известного разбиения точек поверхности на 4 типа.

ОПРЕДЕЛЕНИЕ 2. Точка поверхности называется эллиптической, если вторая квадратичная форма поверхности в этой точке является знакоопределенной; точка называется гиперболической, если вторая квадратичная форма поверхности в этой точке не является знакоопределенной и невырожденна; точка называется параболической, если вторая квадратичная форма поверхности в этой точке вырожденна, но ненулевая; точка называется вырожденной, если вторая квадратичная форма поверхности в этой точке нулевая.

Bce 22 типа точек коориентированных поверхностей описаны в теореме 1 и табл. 1 . В столбце $k$ располагаются типы 3 -струй, или типы точек, заполняющих на поверхности общего положения множества коразмерности $k$. Так, в столбце 0 содержатся типы точек, заполняющих на поверхности общего положения открытые области, в столбце 7 содержатся типы точек, встречающиеся лишь в 5 -параметрических семействах поверхностей.

ЗАмЕчАНИЕ. Если вблизи эллиптической точки поверхность и коориентирующая нормаль лежат по одну сторону от касательной плоскости, то точка называется положительной и ей приписывается нижний индекс + . Аналогичным образом определяется и знак параболической точки. Положительные гиперболические точки граничат на поверхности с положительными эллиптическими точками, а

* Работа частично поддержана Российским фондом фундаментальных исследований (проект 9901-01109). 
отрицательные - с отрицательными. Тип точек $D_{4}^{+}$и $D_{4}^{-}$не меняется при смене коориентации поверхности.

Таблица 1

\begin{tabular}{|l||c|c|c|c|c|c|c|c|}
\hline & 0 & 1 & 2 & 3 & 4 & 5 & 6 & 7 \\
\hline \hline эллиптические & $E_{ \pm}$ & & $S E_{ \pm}$ & & & & & \\
\hline гиперболические & $H_{ \pm}$ & $I_{l}, I_{r}$ & $S H$ & & & & & \\
\hline параболические & & $P_{ \pm}$ & $S P_{ \pm}$ & $A_{ \pm}^{4}$ & $s_{ \pm}$ & & & \\
\hline вырожденные & & & & $D_{4}^{ \pm}$ & $q_{1}$ & $q_{2}$ & & $q_{3}$ \\
\hline
\end{tabular}

ЗАмЕЧАНИЕ. Типы 3-струй поверхностей, входящие в первые три столбца табл. 1 (за исключением специальных эллиптических точек $S E_{ \pm}$) встречались в работе [2] в контексте проектирований двумерных поверхностей.

Группа аффинных преобразований трехмерного пространства действует на пространстве 3-струй коориентированных поверхностей. При этом пространство 3 струй разбивается на 22 орбиты. Различные орбиты соответствуют различным типам точек поверхностей. Чтобы получить классификацию орбит, достаточно ограничиться изучением следующей подгруппы группы аффинных преобразований.

ОПРЕДЕЛЕНИЕ 3. Рассмотрим трехмерное аффинное пространство с фиксированной системой координат $(x, y, z)$. Семимерная группа линейных преобразований этого пространства, сохраняющих ориентацию пространства и переводящих полупространство $z \geqslant 0$ в себя, называется малой аффинной группой и обозначается через $\mathrm{Aff}_{7}$. Пространство всех $k$-струй поверхностей, касающихся плоскости $z=0$ в точке $(0,0,0)$, называется малым пространством $k$-струй и обозначается через $J_{k}$.

ЗАмЕЧАНИЕ. Для любой поверхности, касающейся плоскости $z=0$ в точке $(0,0,0)$, ее $k$-струя в точке $(0,0,0)$ единственным образом представляется как $k$-струя поверхности вида $z=P_{2}+\cdots+P_{k}$, где $P_{i}-$ однородный многочлен степени $i$ от переменных $(x, y)$. Поэтому в дальнейшем точки пространства $J_{k}$ всегда будут отождествляться с наборами из $k-1$ однородных многочленов.

ТеоремА 1. Пространство $J_{3}$ разбивается под действием группь Aff 7 на 22 орбитьл. В следующем списке приведено по одному представителю орбит коразмерностей $0-4$ :

(1) открытые орбитьь

$$
E_{ \pm} \ni\left( \pm\left(x^{2}+y^{2}\right)+x^{3}\right), \quad H_{ \pm} \ni\left( \pm\left(x^{2}-y^{2}\right)+x^{3}\right)
$$

(2) орбиты коразмерности 1

$$
P_{ \pm} \ni\left( \pm y^{2}+x^{3}\right), \quad I_{l} \ni\left(x y+x^{3}\right), \quad I_{r} \ni\left(-x y+x^{3}\right)
$$

(3) орбиты коразмерности 2

$$
S E_{ \pm} \ni \pm\left(x^{2}+y^{2}\right), \quad S H \ni\left(x^{2}-y^{2}\right), \quad S P_{ \pm} \ni\left( \pm x^{2}+x y^{2}\right) ;
$$

(4) орбиты коразмерности 3

$$
A_{ \pm}^{4} \ni\left( \pm x^{2}+x^{2} y\right), \quad D_{4}^{ \pm} \ni x\left(x^{2} \pm y^{2}\right) ;
$$


(5) орбиты коразмерности 4

$$
s_{ \pm} \ni\left( \pm x^{2}\right), \quad q_{1} \ni x^{2}(x+y) .
$$

Многие из типов точек, перечисленных в теореме 1 , допускают простые геометрические определения. Точки орбит $I_{l}$ и $I_{r}-$ это точки поверхности, в которых асимптотическая линия имеет нулевую кривизну. Специальные эллиптические точки $S E_{ \pm}-$это такие точки, в которых 3 -струю поверхности можно породить эллипсоидом. В специальных гиперболических точках 3-струю поверхности можно породить однополостным гиперболоидом. Таким образом, эллипсоиды состоят целиком из специальных эллиптических точек, а однополостные гиперболоиды - из специальных гиперболических точек. Точки орбит $A_{ \pm}^{4}, D_{4}^{ \pm}$соответствуют перестройкам (рождениям и уничтожениям) специальных эллиптических, гиперболических и параболических точек.

Bce орбиты действия группы $\mathrm{Aff}_{7}$ являются ориентируемыми (открытыми) многообразиями; в \$3 будут выбраны ориентации всех орбит. Для пары ориентированных орбит соседней размерности стандартным образом определяется кратность примыкания (см. [1]). Автоматически возникает и определение границы орбиты. Получающийся комплекс называется универсальным комплексом особенностей [1]. В следующей теореме выписаны границы орбит коразмерностей 0-4 действия группы $\mathrm{Aff}_{7}$ (относительно ориентации из §3).

Теорема 2. Имеют место следующие соотношения:

$$
\begin{aligned}
& \partial\left(s_{ \pm}\right)=q_{2}, \quad \partial\left(D_{4}^{+}\right)=q_{1}, \quad \partial\left(S E_{+}\right)=D_{4}^{+}+A_{+}^{4}, \\
& \partial\left(q_{1}\right)=0, \quad \partial\left(D_{4}^{-}\right)=-q_{1}, \quad \partial\left(S E_{-}\right)=-\left(D_{4}^{+}+A_{+}^{4}\right), \\
& \partial\left(A_{+}^{4}\right)=-q_{1}, \quad \partial\left(S P_{+}\right)=2 A_{+}^{4}-D_{4}^{+}-3 D_{4}^{-}, \\
& \partial\left(A_{-}^{4}\right)=-q_{1}, \quad \partial\left(S P_{-}\right)=-2 A_{-}^{4}+D_{4}^{+}+3 D_{4}^{-}, \\
& \partial(S H)=A_{-}^{4}-A_{+}^{4}, \\
& \partial\left(I_{l}\right)=S P_{+}+S P_{-}+2 S H, \\
& \partial\left(H_{+}\right)=I_{l}-I_{r}-P_{+}, \\
& \partial\left(I_{r}\right)=S P_{+}+S P_{-}+2 S H, \\
& \partial\left(H_{-}\right)=-I_{l}+I_{r}+P_{-}, \\
& \partial\left(P_{+}\right)=0 \text {, } \\
& \partial\left(E_{+}\right)=P_{+} \text {, } \\
& \partial\left(P_{-}\right)=0 \text {, } \\
& \partial\left(E_{-}\right)=-P_{-} \text {. }
\end{aligned}
$$

СледСтвиЕ. Суммы $S P_{+}+S P_{-}+2 S H, S E_{+}+S H+S E_{-}$орбит коразмерности два являются цииклами.

В §2 будет указана естественная конструкция, позволяющая каждой специальной эллиптической, гиперболической и параболической точке поверхности сопоставить некоторый индекс (в случае общего положения это \pm 1 ). Этот индекс не зависит от коориентации поверхности. Соотношения между числами специальных точек компактной поверхности, подсчитанными с индексами, устанавливаются в следующей теореме.

ТЕОремА 3. Пусть задана иммерсия общего положения замкнутой поверхности $M$ рода g в пространство $\mathbb{R}^{3}$. Тогда для чисел se, , sh, sp эллиптических, гиперболических и параболических точек на этой поверхности, подсчитанных с индексами, верны следующие тождества:

$$
s p_{+}+s p_{-}+2 s h=0, \quad s e_{+}+s h+s e_{-}=6 g-6 .
$$


ЗАмЕЧАНИЕ. Утверждение теоремы верно и для иммерсий общего положения замкнутых поверхностей в пространство $\mathbb{R} \mathbb{P}^{3}$.

Известно, что кубическая поверхность общего положения, диффеоморфная проективной плоскости, содержит ровно четыре параболические кривые и шесть специальных параболических точек [3]. Кроме того, эта поверхность содержит ровно три вещественные проективные прямые. Специальные параболические точки являются точками касания указанных трех прямых и четырех параболических кривых. Е. И. Шустин показал (см. [4]), что распределение специальных параболических точек по четырем параболическим кривым таково: $6=0+1+2+3$. В следующих двух теоремах рассматриваются лишь кубические поверхности общего положения (необходимо, чтобы комплексификация поверхности содержала 27 различных проективных прямых, пересекающихся лишь попарно).

Tеорема 4. Рассмотрим в трехмерном проективном пространстве $\mathbb{R P}^{3} \kappa y$ бическую поверхность, диффеоморфную двумерной проективной плоскости. Такая поверхность содержит три специальные гиперболические точки и шесть специальных эллиптических точек. Для любой параболической кривой на этой поверхности сумма числа специильных точек на этой кривой и числа спещиальньх эллиптических точек внутри кривой равна трем. Проективная плоскость, касательная $\kappa$ такой поверхности в любой специальной эллиптической точке, пересекается с поверхностью по проективной прямой. Специиальые гиперболические точки поверхности являются точками пересечения трех проективных прямых, лежащих на поверхности.

Картина кубической поверхности, описанная в теореме 4 , изображена на рис. 1. С точностью до симметрии (здесь действует симметрическая группа $S_{4}$ ) это единственная реализуемая картина кубической поверхности общего положения, диффеоморфной проективной плоскости.

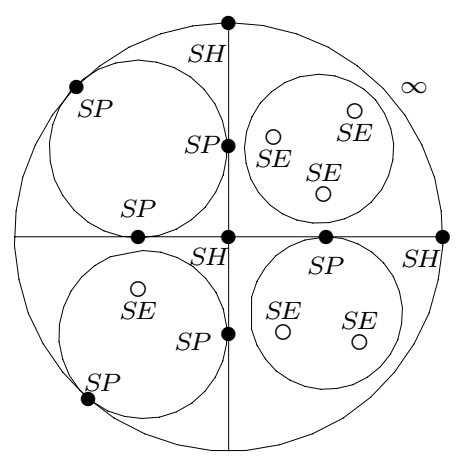

Рис. 1

Tеорема 5. Пусть кубическая поверхность состоит из двух компонент связности. В этом случае одна из компонент диффеоморфна сфере (другая - проективной плоскости) и на этой сфере лежит ровно шесть специальных эллиптических точек.

ЗАмЕчАНиЕ. Комплексная кубическая поверхность содержит ровно 27 проективных прямых. Эти 27 прямых можно увидеть и на вещественной кубической поверхности, состоящей из двух компонент связности. Три прямые целиком лежат 
на компоненте, диффеоморфной проективной плоскости, остальные 24 проявляются в виде специальных эллиптических точек. Шесть точек лежат на сферической компоненте, шесть на проективной и через каждую точку проходят две комплексно-сопряженные прямые.

B §1 проводится классификация орбит действия группы Aff 7 на пространстве $J_{3}$ и указываются некоторые простейшие свойства орбит. В $\$ 2$ изучается действие различных симметрий на пространстве $J_{3}$ и доказываются некоторые технические леммы, необходимые для теоремы 2. В $\$ 3$ доказываются теоремы 2 и 3 . В $\$ 4$ доказываются теоремы 4 и 5.

Выражаю признательность В. И. Арнольду и М. Э. Казаряну за интерес к работе и полезные комментарии.

\section{§0. Простейшие примеры}

В этом параграфе будут описаны реализации всех типов 3-струй, содержащихся в первых четырех столбцах табл. 1.

ПримеР 1. Рассмотрим в пространстве $\mathbb{R}^{3}$ следующее однопараметрическое семейство поверхностей:

$$
z=x^{3}-3 x y^{2}+t\left(x^{2}+y^{2}\right) .
$$

На рис. 2 изображены стратификации поверхностей семейства для $t<0, t=0$, $t>1$ (естественно, рассматривается проекция поверхности на плоскость $z=0$ ). Точки типа $I$ образуют на поверхности три прямые, пересекающиеся под углами $60^{\circ}$. При $t \neq 0$ три точки пересечения этих прямых являются специальными гиперболическими точками. Множество параболических точек образует окружность радиуса $|t| / 3$, причем точки касания этой окружности с тремя указанными прямыми являются специальными параболическими. При $t=0$ параболическая кривая схлопывается в точку и происходит перестройка орбиты $D_{4}^{-}$.
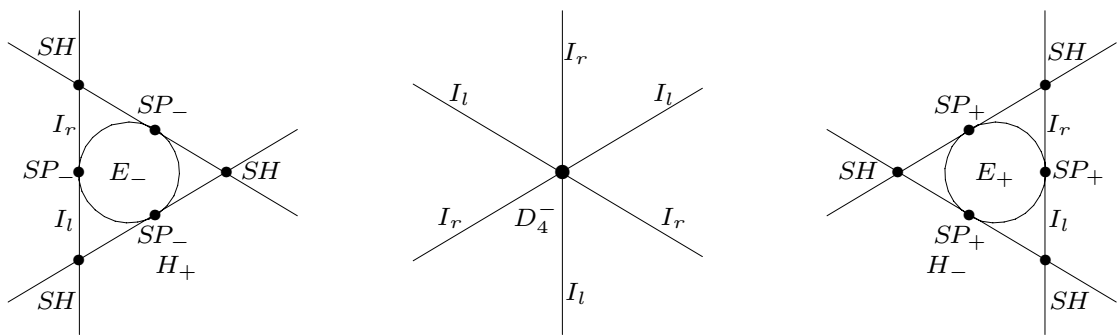

Рис. 2

Пример 2. Рассмотрим в пространстве $\mathbb{R}^{3}$ следующее однопараметрическое семейство поверхностей:

$$
z=x^{3}+3 x y^{2}+t\left(x^{2}+y^{2}\right) .
$$

Точки типа $I$ образуют на каждой поверхности семейства прямую, касающуюся параболической кривой (гиперболы при $t \neq 0$ ) в параболической точке. Вместо трех специальных параболических точек присутствует одна специальная эллиптическая точка. При $t=0$ в этом семействе происходит перестройка орбиты $D_{4}^{+}$ (рис. 3). 

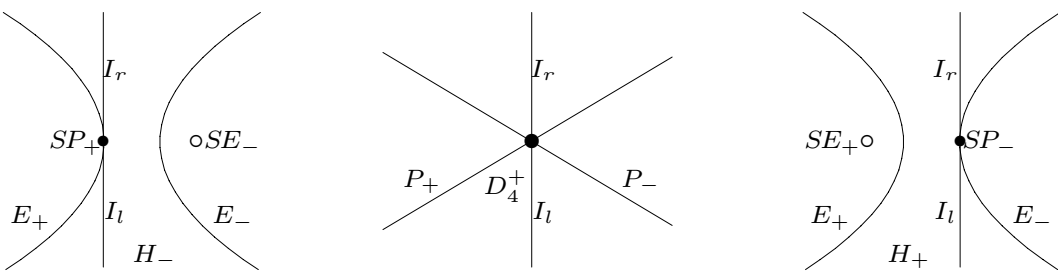

Рис. 3

На рис. 4 изображена картина, возникающая на поверхности при возмущении точки типа $A_{ \pm}^{4}$.
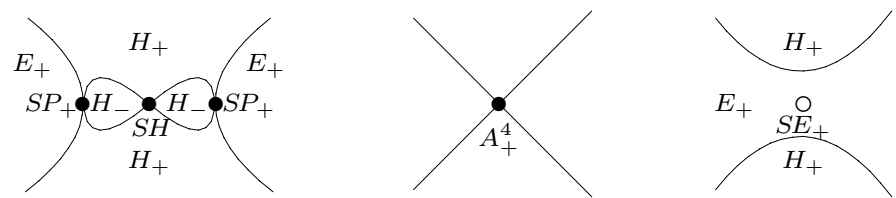

Рис. 4

\section{§1. Орбиты действия группы $\mathrm{Aff}_{7}$ на пространстве $J_{3}$}

Имеет место очевидное расслоение $J_{3} \rightarrow J_{2}$, и действие группы $\mathrm{Aff}_{7}$ сохраняет структуру этого расслоения. Каждый из слоев этого расслоения целиком состоит из точек лишь одного типа - эллиптического, гиперболического, параболического или вырожденного. Орбиты действия группы Aff 7 , пересекаясь со слоями расслоения, задают их стратификации. При этом стратификации всех эллиптических слоев (соответственно гиперболических, параболических, вырожденного) диффеоморфны. Классификация орбит действия группы Aff $_{7}$ на пространстве $J_{3}$ фактически заключается в описании стратификаций слоев различных типов.

Рассмотрим в пространстве $J_{3}$ четыре слоя $e, h, p, d$, состоящие из точек вида $x^{2}+y^{2}+P_{3}, x y+P_{3}, x^{2}+P_{3}$ и $P_{3}$. Для каждого из них найдем максимальную подгруппу группы $\mathrm{Aff}_{7}$, для которой данный слой является инвариантным. Эти группы обозначим через $E, H, P, D$. В предложении 1 перечисляются орбиты действия групп $E, H, P$ и $D$ на соответствующих слоях. Введем во всех слоях координаты $t_{1}, t_{2}, t_{3}, t_{4}$, равные коэффициентам при членах $x^{3}, x^{2} y, x y^{2}, y^{3}$ многочлена $P_{3}$.

ПрЕДЛОЖЕниЕ 1. (1) Действие группь Е на слое е имеет ровно две орбить.

(2) Действие группь $H$ на слое $h$ имеет ровно девять орбит.

(3) Действие группы $P$ на слое р имеет ровно семь орбит.

(4) Действие группы $D$ на слое

ДоказАТЕЛьство. (1) Эллиптические точки. Алгебра Ли группы $E$ естественно отображается в алгебру векторных полей на слое $e$. Найдем это отображение. Выберем в алгебре Ли группы $E$ следующий базис:

$$
v_{1}=z \frac{\partial}{\partial x}, \quad v_{2}=z \frac{\partial}{\partial y}, \quad v_{3}=-\left(2 z \frac{\partial}{\partial z}+x \frac{\partial}{\partial x}+y \frac{\partial}{\partial y}\right), \quad v_{4}=x \frac{\partial}{\partial y}-y \frac{\partial}{\partial x} .
$$


Полям $v_{1}, v_{2}, v_{3}, v_{4}$ соответствуют следующие векторные поля на слое $e$ :

$$
\begin{gathered}
v_{1}^{\prime}=-2\left(\frac{\partial}{\partial t_{1}}+\frac{\partial}{\partial t_{3}}\right), \quad v_{2}^{\prime}=-2\left(\frac{\partial}{\partial t_{2}}+\frac{\partial}{\partial t_{4}}\right), \\
v_{3}^{\prime}=t_{1} \frac{\partial}{\partial t_{1}}+t_{2} \frac{\partial}{\partial t_{2}}+t_{3} \frac{\partial}{\partial t_{3}}+t_{4} \frac{\partial}{\partial t_{4}}, \\
v_{4}^{\prime}=-t_{2} \frac{\partial}{\partial t_{1}}+\left(3 t_{1}-2 t_{3}\right) \frac{\partial}{\partial t_{2}}+\left(2 t_{2}-3 t_{4}\right) \frac{\partial}{\partial t_{3}}+t_{3} \frac{\partial}{\partial t_{4}} .
\end{gathered}
$$

Точки слоя, в которых поля $v_{1}^{\prime}, \ldots, v_{4}^{\prime}$ линейно зависимы, задаются уравнением $\left(t_{1}-t_{3}\right)^{2}+\left(t_{2}-t_{4}\right)^{2}=0$. В дополнении к плоскости $t_{1}=t_{3}, t_{2}=t_{4}$ ранг системы векторных полей $v_{1}^{\prime}, \ldots, v_{4}^{\prime}$ равен 4 ; поэтому дополнение является орбитой действия группы $E$. Поля $v_{1}^{\prime}$ и $v_{2}^{\prime}$ касаются плоскости $t_{1}=t_{3}, t_{2}=t_{4}$ и порождают группу сдвигов этой плоскости; поэтому плоскость $t_{1}=t_{3}, t_{2}=t_{4}$ является орбитой действия группы $E$.

(2) Гиперболические точки. Будем действовать по той же схеме, что и в предыдущем пункте. Выберем в алгебре Ли группы $H$ следующий базис:

$$
v_{1}=z \frac{\partial}{\partial x}, \quad v_{2}=z \frac{\partial}{\partial y}, \quad v_{3}=-\left(2 z \frac{\partial}{\partial z}+x \frac{\partial}{\partial x}+y \frac{\partial}{\partial y}\right), \quad v_{4}=x \frac{\partial}{\partial x}-y \frac{\partial}{\partial y} .
$$

Полям $v_{1}, v_{2}, v_{3}, v_{4}$ соответствуют следующие векторные поля на слое $h$ :

$$
\begin{gathered}
v_{1}^{\prime}=-\frac{\partial}{\partial t_{3}}, \quad v_{2}^{\prime}=-\frac{\partial}{\partial t_{2}}, \quad v_{3}^{\prime}=t_{1} \frac{\partial}{\partial t_{1}}+t_{2} \frac{\partial}{\partial t_{2}}+t_{3} \frac{\partial}{\partial t_{3}}+t_{4} \frac{\partial}{\partial t_{4}}, \\
v_{4}^{\prime}=-3 t_{1} \frac{\partial}{\partial t_{1}}-t_{2} \frac{\partial}{\partial t_{2}}+t_{3} \frac{\partial}{\partial t_{3}}+3 t_{4} \frac{\partial}{\partial t_{4}} .
\end{gathered}
$$

Точки слоя, в которых поля $v_{1}^{\prime}, \ldots, v_{4}^{\prime}$ линейно зависимы, задаются уравнением $t_{1}=0$ или $t_{4}=0$. Поэтому имеются ровно 4 открытые орбиты действия группы $H$ на слое - дополнение к объединению гиперплоскостей. Кроме того, имеются 4 орбиты коразмерности $1-$ полуплоскости, на которые плоскость $t_{1}=t_{4}=0$ делит гиперплоскости $t_{1}=0$ и $t_{4}=0$. Плоскость $t_{1}=t_{4}=0$ также является орбитой.

(3) Параболические точки. Выберем в алгебре Ли группы $P$ такой базис:

$$
v_{1}=z \frac{\partial}{\partial x}, \quad v_{2}=z \frac{\partial}{\partial y}, \quad v_{3}=-\left(2 z \frac{\partial}{\partial z}+x \frac{\partial}{\partial x}+y \frac{\partial}{\partial y}\right), \quad v_{4}=x \frac{\partial}{\partial y}, \quad v_{5}=y \frac{\partial}{\partial y} .
$$

Полям $v_{1}, v_{2}, v_{3}, v_{4}, v_{5}$ соответствуют следующие векторные поля на слое $p$ :

$$
\begin{gathered}
v_{1}^{\prime}=-2 \frac{\partial}{\partial t_{1}}, \quad v_{2}^{\prime}=0, \quad v_{3}^{\prime}=t_{1} \frac{\partial}{\partial t_{1}}+t_{2} \frac{\partial}{\partial t_{2}}+t_{3} \frac{\partial}{\partial t_{3}}+t_{4} \frac{\partial}{\partial t_{4}}, \\
v_{4}^{\prime}=-t_{2} \frac{\partial}{\partial t_{1}}-2 t_{3} \frac{\partial}{\partial t_{2}}-3 t_{4} \frac{\partial}{\partial t_{3}}, \quad v_{5}^{\prime}=-t_{2} \frac{\partial}{\partial t_{2}}-2 t_{3} \frac{\partial}{\partial t_{3}}-3 t_{4} \frac{\partial}{\partial t_{4}} .
\end{gathered}
$$

Слой $p$ содержит полный флаг пространств, инвариантных относительно действия группы $P$. Это следующие пространства: $t_{4}=0, t_{3}=t_{4}=0, t_{2}=t_{3}=$ $t_{4}=0$. Флаг задает разбиение пространства на семь орбит - две открытые, две коразмерности 1, две коразмерности 2 и одну коразмерности 3.

(4) Bырожденные точки. Действие группы $\mathrm{Aff}_{7}$ на пространстве пар $\left(0, P_{3}\right)$ сводится к действию двумерной аффинной группы на пространстве однородных 
многочленов третьей степени. Очевидно, что последнее действие имеет 4 орбиты.

Заметим, что группы $E$ и $H$ являются двумерными аффинными группами.

ДОКАЗАТЕЛЬСТВО тЕОРЕмы 1. В предложении 1 описана картина, высекаемая орбитами действия группы $\mathrm{Aff}_{7}$ на слоях расслоения $J_{3}$. Осталось лишь рассмотреть в совокупности все эллиптические, гиперболические и параболические слои (вырожденный слой единствен).

(1) Эллиптические орбиты. Эллиптический слой пересекается с орбитами действия группы $\mathrm{Aff}_{7}$ по двум стратам размерности 2 и 4 (предложение 1). Любые два положительных эллиптических слоя могут быть переведены друг в друга действием группы $\mathrm{Aff}_{7}$. Поэтому существует ровно две положительные эллиптические орбиты размерностей 5 и 7.

(2) Гиперболические орбиты. Орбиты действия группы Aff 7 высекают из гиперболического слоя четыре открытых страта - дополнение к паре гиперплоскостей. При монодромии слоя, порождаемой поворотом плоскости $(x, y)$ на $180^{\circ}$, противоположные страты меняются местами. Поэтому существуют ровно две отрытые гиперболические орбиты действия группы Aff $_{7}$ на пространстве $J_{3}$. Аналогично показывается, что есть ровно две гиперболические орбиты коразмерности 1, и автоматически получается, что есть ровно одна орбита коразмерности 2.

(3) Параболические орбиты. Разбор этого случая аналогичен предыдущему.

Теорема доказана.

В следующем предложении дано описание восемнадцати орбит действия группы $\mathrm{Aff}_{7}$ в терминах корней пар многочленов $\left(P_{2}, P_{3}\right)$.

ПрЕДЛОЖЕНИЕ 2. (1) $E_{+}\left(E_{-}\right)$. Многочлен $P_{2}$ положительно (отрицательно) определен и не имеет общих корней с многочленом $P_{3}$.

(2) $\mathrm{SE}_{+}\left(S E_{-}\right)$. Многочлен $P_{2}$ положительно (отрицательно) определен, $u$ многочлен $P_{3}$ кратен $P_{2}$.

(3) $H_{+}\left(H_{-}\right)$. Многочлен $P_{2}$ гиперболичен, и в положительном секторе многочлена $P_{2}$ лежит нечетное (четное) число нулей многочлена $P_{3}$. Кроме того, $P_{2}$ не имеет общих корней с $P_{3}$.

(4) $P_{+}\left(P_{-}\right)$. Многочлен $P_{2}$ положительно (отрицательно) определен и не имеет обиих корней с многочленом $P_{3}$.

(5) $I_{r}\left(I_{l}\right)$. Многочлен $P_{2}$ гиперболичен и на правой (левой) границе его положительного сектора лежит корень многочлена $P_{3}$. Многочлень $P_{2}$ и $P_{3}$ имеют ровно один общий корень.

(6) $S P_{+}\left(S P_{-}\right)$. Многочлен $P_{2}$ параболический и неотрицателен (неположителен), а многочлен $P_{3}$ имеет ровно один общий корень с многочленом $P_{2}$.

(7) $A_{+}^{4}\left(A_{-}^{4}\right)$. Многочлен $P_{2}$ параболический и неотрицателен (неположителен), а многочлен $P_{3}$ имеет двойной корень и кратен $P_{2}$.

(8) $D_{4}^{+}\left(D_{4}^{-}\right)$. Многочлен $P_{2}$ нулевой, а многочлен $P_{3}$ имеет 1 вещественный корень (3 вещественных корня).

(9) $s_{+}\left(s_{-}\right)$. Многочлен $P_{2}$ параболический и неотрицателен (неположителен), а многочлен $P_{3}$ имеет тройной корень и кратен $P_{2}$.

Следующую элементарную лемму приведем без доказательства.

ЛЕмма 1. Пусть связная группа Ли действует на некотором многообразии. Орбита этого действия неориентируема тогда и только тогда, когда существует элемент группь, оставляющий неподвижной одну из точек орбить и меняющий в этой точке ориентацию касательного пространства к орбите. 
СледствиЕ. Все орбиты действия группь $\mathrm{Aff}_{7}$ на пространстве $J_{3}$ являются ориентируемыли (открытылми) многообразиями.

ДокАЗАТЕльство. Выведем из леммы 1 ориентируемость орбиты $S H$. Рассмотрим точку $x y$ орбиты $S H$. Стабилизатор этой точки является абелевой группой, состоящей из двух компонент связности. Компонента, содержащая 1, не может менять ориентацию касательного пространства к орбите $S H$ в точке $x y$. Симметрия плоскости $(x, y) \rightarrow(-x,-y)$ принадлежит второй компоненте связности стабилизатора. Простое вычисление показывает, что и это отображение не меняет ориентации в касательном пространстве к $S H$ в точке $x y$. Осталось применить лемму 1, и ориентируемость орбиты $S H$ доказана. Аналогичным образом доказывается ориентируемость всех остальных орбит.

\section{§2. Кратности примыкания орбит и симметрии}

Для вычисления кратностей примыкания различных орбит введем вспомогательное понятие геометрической кратности примыкания. Для определения этой кратности ориентации орбит не играют роли.

Пусть $\mathrm{O}_{1}$ и $\mathrm{O}_{2}$ - две орбиты действия группы $\mathrm{Aff}_{7}$, причем коразмерность орбиты $\mathrm{O}_{2}$ на единицу больше коразмерности орбиты $O_{1}$. Возьмем произвольную точку орбиты $\mathrm{O}_{2}$ и проведем трансверсаль к этой орбите. Предположим, что трансверсаль пересекает орбиту $O_{1}$ по нескольким гладким лучам.

ОПрЕДЕЛЕНИЕ 4. Число лучей, по которым трансверсаль к орбите $O_{2}$ пересекает орбиту $O_{1}$, называется геометрической кратностью примыккания орбиты $O_{1} \mathrm{~K}$ орбите $\mathrm{O}_{2}$.

В следующем предложении записаны все геометрические кратности примыкания.

ПрЕДЛОЖЕНИЕ 3. Имеют место следующие соотношения:

(1) $\left[E_{ \pm}: P_{ \pm}\right]_{g}=1,\left[H_{ \pm}: P_{ \pm}\right]_{g}=1,\left[H_{ \pm}: I_{l}\right]_{g}=1,\left[H_{ \pm}: I_{r}\right]_{g}=1$;

(2) $\left[I_{l}: S H\right]_{g}=2,\left[I_{l}: S P_{ \pm}\right]_{g}=1,\left[I_{r}: S H\right]_{g}=2,\left[I_{r}: S P_{ \pm}\right]_{g}=1,\left[P_{ \pm}: S P_{ \pm}\right]_{g}=2$;

(3) $\left[S E_{ \pm}: D_{4}^{+}\right]_{g}=1,\left[S E_{ \pm}: A_{ \pm}^{4}\right]_{g}=1,\left[S P_{ \pm}: A_{ \pm}^{4}\right]_{g}=2,\left[S P_{ \pm}: D_{4}^{-}\right]_{g}=3$, $\left[S P_{ \pm}: D_{4}^{+}\right]_{g}=1,\left[S H: A_{ \pm}^{4}\right]_{g}=1,\left[S H: D_{4}^{-}\right]_{g}=6$;

(4) $\left[D_{4}^{ \pm}: q_{1}\right]_{g}=1,\left[A_{ \pm}^{4}: q_{1}\right]_{g}=1,\left[A_{ \pm}^{4}: s_{ \pm}\right]_{g}=2$.

ДокАЗАТЕЛьство. Найдем геометрическую кратность примыкания орбиты $S H$ к орбите $D_{4}^{-}$. Точка $x^{3}-x y^{2}$ лежит на орбите $D_{4}^{-}$. Орбита $D_{4}^{-}$имеет коразмерность 3 , и трансверсаль в точке $x^{3}-x y^{2}$ можно выбрать в следующем виде: $a x^{2}+b x y+c y^{2}+x^{3}-x y^{2}$. Очевидно, что орбита $S H$ пересекается с трансверсалью по шести лучам. Аналогично вычисляются и остальные кратности, фигурирующие в предложении. Предложение доказано.

Рассмотрим в трехмерном пространстве инволюцию $(x, y, z) \rightarrow(x,-y,-z)$. Эта инволюция порождает на пространстве $J_{3}$ некоторую симметрию $\sigma$. В следующем предложении описано действие симметрии $\sigma$ на различных орбитах.

ПрЕДЛОЖЕНИЕ 4. При симметрии б орбиты ведут себя следующим образом:

(0) орбить $H_{ \pm}$переставляются, орбиты $E_{ \pm}$переставляются;

(1) орбиты $I_{l}, I_{r}$ переходят в себя и меняют ориентацию, орбить $P_{ \pm}$ переставляются;

(2) орбита $S H$ переходит в себя и меняет ориентацию, орбить $S P_{ \pm}$nереставляются, орбить $S E_{ \pm}$также переставляются; 
(3) орбить $D_{4}^{ \pm}$сохраняют ориентацию, орбиты $A_{ \pm}^{4}$ переставляются;

(4) орбита $q_{1}$ сохраняет ориентациию, орбиты $s_{ \pm}$переставляются.

ДокАЗАТЕЛЬство. Покажем, что орбита $S H$ меняет ориентацию под действием симметрии $\sigma$. Точка $x y$ принадлежит орбите $S H$ и является неподвижной относительно симметрии $\sigma$. Плоскость, ортогональная к орбите в точке $x y$, порождается векторами $v_{1}=x^{3}, v_{2}=y^{3}$. Симметрия $\sigma$ меняет ориентацию этой плоскости. А значит, и орбита $S H$ обязана изменить ориентацию, так как симметрия $\sigma$ сохраняет ориентацию пространства $J_{3}$. Остальные утверждения предложения доказываются еще проще.

\section{§3. Доказательство теорем 2,3}

При доказательстве теоремы 2 мы будем выбирать ориентации на орбитах таким образом, чтобы выполнялись условия (a), (b), (с) следующей леммы.

Лемма 2. Ориентации орбит действия группь Aff $_{7}$ на пространстве $J_{3}$ можно выбрать так, чтобы выполнялись следующие условия:

(а) сумма орбит $D_{4}^{+}+D_{4}^{-}$является циклом;

(b) сумма орбит $E_{+}+H_{+}+H_{-}+E_{-}$является ициклом;

(c) $\sigma\left(A_{+}^{4}\right)=A_{-}^{4}, \sigma\left(S P_{+}\right)=-S P_{-}, \sigma\left(S E_{+}\right)=-S E_{-}, \sigma\left(P_{+}\right)=-P_{-}$.

ДокАЗАТЕЛЬство. Лемма вытекает из следующих трех фактов: замыкание объединения орбит $D_{4}^{+}$и $D_{4}^{-}$диффеоморфно $\mathbb{R}^{4}$; замыкание объединения орбит $E_{+}$, $H_{+}, H_{-}$и $E_{-}$диффеоморфно $\mathbb{R}^{7}$; симметрия $\sigma$ переставляет пары орбит $A_{+}^{4}$ и $A_{-}^{4}, S P_{+}$и $S P_{-}, S E_{+}$и $S E_{-}, P_{+}$и $P_{-}$.

Лемма 3. Пусть орбиты действия группь Aff 7 на пространстве $J_{3}$ ориентированы так, ито условия (a), (b), (c) леммь 2 удовлетворяются. Тогда симметрия $\sigma$ и дифференциал д коммутируют.

ДОКАЗАТЕЛЬСТВО. При выПолнении условий (a), (b), (c) симметрия $\sigma$ одинаково действует на орбитах одной коразмерности, сохраняет ориентации орбит коразмерностей $0,3,4,5$ и меняет ориентации орбит коразмерностей 1,2 .

Лемма 4. (1) Кратность примыкания орбиты $S P_{+} \kappa$ орбите $D_{4}^{-}$по модулю равняется трем.

(2) Пусть орбиты $D_{4}^{ \pm}$ориентированы согласно условию (а). Тогда кратности примыкания орбиты $S P_{+} \kappa$ орбитам $D_{4}^{-}$и $D_{4}^{+}$имеют один и тот же знак.

ДокАЗАтельство. (1) Рассмотрим точку $x^{3}-3 x y^{2}$, лежащую на орбите $D_{4}^{-}$, и проведем трансверсаль, состоящую из точек вида $x^{3}-3 x y^{2}+a x^{2}+b x y+c y^{2}$. Трансверсаль пересекает орбиту $S P_{+}$по трем лучам. Покажем, что каждый из трех лучей вносит одинаковый вклад в кратность примыкания орбиты $S P_{+}$к $D_{4}^{-}$. Для этого достаточно указать группу из трех элементов, действующую на пространстве $J_{3}$, оставляющую неподвижной точку $x^{3}-3 x y^{2}$, сохраняющую ориентации орбит $S P_{+}, D_{4}^{-}$и циклически переставляющую три луча, входящие в точку $x^{3}-3 x y^{2}$. Группа поворотов плоскости $(x, y)$ на углы, кратные $2 \pi / 3$, удовлетворяет всем перечисленным требованиям. Первая половина леммы доказана.

(2) Рассмотрим путь $x\left(x^{2}+x y+t y^{2}\right), t \in[-1,1]$, лежащий в замыкании объединения орбит $D_{4}^{+}$и $D_{4}^{-}$. Для всех точек этого пути выберем трансверсаль в виде $x\left(x^{2}+x y+t y^{2}\right)+a x^{2}+b x y+c y^{2}$. При любом значении $t$ среди лучей, высекаемых трансверсалью из орбиты $S P_{+}$, присутствует луч $x\left(x^{2}+x y+t y^{2}\right)+$ 
$a x^{2}, a>0$. Понятно, что этот луч вносит один и тот же вклад в кратность примыкания орбиты $S P_{+}$во всех точках пути. Осталось воспользоваться первой половиной леммы. Лемма доказана.

ДОКАЗАТЕЛЬСТВО ТЕОРЕМЫ 2. МЫ Последовательно будем вводить ориентации различных орбит, одновременно доказывая теорему 2. При этом из восьми орбит $A_{+}^{4}, S P_{+}, S E_{+}, P_{+}, A_{-}^{4}, S P_{-}, S E_{-}, P_{-}$нам будет достаточно определить ориентацию лишь первых четырех, остальные будут ориентированы согласно условию (c).

(1) Выберем сначала ориентацию орбит $D_{4}^{+}$и $q_{1}$ так, чтобы выполнялось равенство $\partial\left(D_{4}^{+}\right)=q_{1}$. Тогда из условия (а) получаем $\partial\left(D_{4}^{-}\right)=-q_{1}$. Орбиту $A_{+}^{4}$ можно ориентировать (предложение 2 ), чтобы выполнялось равенство $\partial\left(A_{+}^{4}\right)=-q_{1}$. Тогда равенство $\partial\left(A_{-}^{4}\right)=-q_{1}$ будет вытекать из (с).

(2) Ориентируем орбиту $S P_{+}$таким образом, чтобы кратность ее примыкания к $D_{4}^{+}$равнялась -1 . Тогда кратность примыкания к $D_{4}^{-}$будет равна -3 (лемма 4). Используя $\partial^{2}\left(S P_{+}\right)=0$, получаем $\partial\left(S P_{+}\right)=2 A_{+}^{4}-D_{4}^{+}-3 D_{4}^{-}$. Из леммы 3 выводим соотношение $\partial\left(S P_{-}\right)=-\sigma\left(\partial\left(S P_{+}\right)\right)=-2 A_{-}^{4}+D_{4}^{+}+3 D_{4}^{-}$.

Ориентируем орбиту $S H$ таким образом, чтобы ее кратность примыкания к $A_{-}^{4}$ равнялась 1. Симметрия $\sigma$ меняет ориентацию орбиты $S H$ и сохраняет ориентации орбит $D_{4}^{ \pm}$; поэтому кратность примыкания орбиты $S H$ к орбитам $D_{4}^{ \pm}$ равна 0. Наконец, из $\sigma(\partial(S H))=-\partial(S H)$ выводим $\partial(S H)=A_{-}^{4}-A_{+}^{4}$.

Орбиту $S E_{+}$ориентируем так чтобы ее кратность примыкания к $A_{+}^{4}$ равнялась 1. Тогда выполняется равенство $\partial\left(S E_{+}\right)=D_{4}^{+}+A_{+}^{4}$, и из леммы 3 следует, что $\partial\left(S E_{-}\right)=-\left(D_{4}^{+}+A_{-}^{4}\right)$.

(3) Введем ориентацию на $I_{l}$ так, чтобы ее кратность примыкания к орбите $S P_{+}$равнялась 1 . Тогда из $\sigma\left(I_{l}\right)=-I_{l}$ и $\partial^{2}\left(I_{l}\right)=0$ вытекает, что $\partial\left(I_{l}\right)=$ $S P_{+}+S P_{-}+2 S H$. Орбиту $I_{r}$ также можно ориентировать таким образом, чтобы выполнялось $\partial\left(I_{r}\right)=S P_{+}+S P_{-}+2 S H$.

(4) Ориентации орбит $H_{ \pm}, E_{ \pm}$выберем согласованно, так чтобы они порождали одну и ту же ориентацию пространства $J_{3}$. При этом потребуем, чтобы кратность примыкания орбиты $H_{+}$к орбите $I_{l}$ равнялась 1 . Орбиту $P_{+}$ориентируем так, чтобы $\partial\left(E_{+}\right)=P_{+}$. Тогда автоматически будут выполняться равенства $\partial\left(H_{+}\right)=I_{l}-I_{r}-P_{+}, \partial\left(H_{-}\right)=-I_{l}+I_{r}+P_{-}, \partial\left(E_{+}\right)=P_{+}, \partial\left(E_{-}\right)=-P_{-}$. Теорема доказана.

Индекс специальной точки. Для определения индекса специальной точки поверхности необходимо фиксировать ориентацию трехмерного пространства, коориентация самой поверхности на индекс специальной точки не влияет.

Пусть $O$ - некоторая специальная гиперболическая (параболическая или эллиптическая) точка поверхности $M$, вложенной в пространство $\mathbb{R P}^{3}$. Построим отображение некоторой окрестности точки $O$ на поверхности $M$ в пространство $J_{3}$ и определим индекс точки $O$ как индекс пересечения образа этой окрестности с орбитой $S H$. Введем в окрестности точки $O$ в $\mathbb{R} \mathbb{P}^{3}$ аффинные координаты $(x, y, z)$, задающие выбранную заранее ориентацию пространства, так чтобы поверхность задавалась уравнением $z=f(x, y), d f(0,0)=0$. Искомое отображение будет сопоставлять точке $\left(x_{0}, y_{0}, f\left(x_{0}, y_{0}\right)\right)$ поверхности $M$ второй и третий член ряда Тейлора функции $f$ в точке $\left(x_{0}, y_{0}\right)$. Будем считать, что ориентация поверхности задается координатами $(x, y)$. 
Покажем, что индекс точки, определенный как индекс пересечения образа окрестности с орбитой $S H$, не зависит от выбора координат $(x, y, z)$. Это утверждение очевидно для двух систем координат, индуцирующих одинаковую ориентацию на поверхности $M$. Рассмотрим в окрестности точки $O$ систему координат $(x,-y,-z)$. Симметрия $\sigma:(x, y, z) \rightarrow(x,-y,-z)$ меняет ориентацию орбиты $S H$ (предложение 2 ) и сохраняет ориентацию самого пространства $J_{3}$. Значит, индекс пересечения образа поверхности $M$ с орбитой $S H$, определенный в координатах $(x, y, z)$ и $(x,-y,-z)$, одинаков (так как координаты $(x,-y)$ и $(x, y)$ задают на $M$ разные ориентации).

ЗАмЕЧАНИЕ. В определении ориентаций орбит действия группы $\mathrm{Aff}_{7}$ содержится произвол, так как орбита $D_{4}^{+}$может быть ориентирована двумя разными способами. Однако этот произвол не влияет на индексы специальных точек, поскольку при смене ориентации орбиты $D_{4}^{+}$меняется ориентация и всех остальных орбит. Согласно этому определению, индексы специальных гиперболических точек, содержащихся в примере $1 \S 0$, равны -1 .

ДокАЗАТЕЛЬСтво теОРЕмы 3. (1) Докажем первое утверждение теоремы 3: $s p_{+}+s p_{-}+2 s h=0$. Пусть задана иммерсия поверхности $M$ в пространство $\mathbb{R}^{3}\left(\mathbb{R P}^{3}\right)$. Введем в рассмотрение пространство $J_{3} M$ всех 3 -струй поверхностей, касающихся образа поверхности $M$. Пространство $J_{3} M$ содержит два особых подмногообразия $N_{1}$ и $N_{2}$, представляющих расслоения над поверхностью $M$ со слоями, изоморфными $S P_{+}+S P_{-}+2 S H$ и $I_{l}$. Из теоремы 2 можно вывести, что $\partial\left(N_{2}\right)=N_{1}$. Таким образом, индекс пересечения любого двумерного цикла с многообразием $N_{1}$ равен нулю. Но сумма $s p_{+}+s p_{-}+2 s h$ как раз и представляет индекс пересечения 3-струйного расширения поверхности $M$ с циклом $N_{1}$.

(2) Рассмотрим теперь в пространстве $J_{3} M$ особое подмногообразие $N_{3}$, представляющее расслоение над поверхностью $M$ со слоями, изоморфными $S E_{+}+S H+S E_{-}$. Равенство $s e_{+}+s h+s e_{-}=6 g-6$ есть утверждение об индексе пересечения сечения расслоения $J_{3} M$ с циклом $N_{3}$. Вычисление этого индекса пересечения будет сведено к вычислению класса Эйлера некоторого двумерного векторного расслоения над поверхностью $M$.

Введем несколько объектов, необходимых для вычисления индекса пересечения. Прежде всего фиксируем метрику в пространстве $\mathbb{R}^{3}$. Это позволит отождествить пространство $J_{3} M$ с $\left(S^{2} \oplus S^{3}\right)\left(T^{*} M\right)$ (прямая сумма симметрического квадрата и куба кокасательного расслоения поверхности $M$ ). В пространстве $\left(S^{2} \oplus S^{3}\right)\left(T^{*} M\right) \quad$ выделим подрасслоение $Q$, состоящее из троек $\left(x, Q_{2}(x), Q_{3}(x)\right)$, где $Q_{2}(x)$ является первой квадратичной формой поверхности, а форма $Q_{3}(x)$ произвольна. Это расслоение имеет структуру векторного расслоения, так как оно изоморфно векторному расслоению $S^{3}\left(T^{*} M\right)$. В расслоении $Q$ выделим двумерное подрасслоение $q$, где форма $Q_{2}(x)$ делит $Q_{3}(x)$.

Рассмотрим, наконец, некоторое сечение $s$ расслоения $J_{3} M$, содержащееся в $Q$. Индекс пересечения $s$ с $N_{3}$ равен индексу пересечения $s$ с $q$, так как $q=$ $Q \cap N_{3}$. Индекс же пересечения $q$ и $s$ равен классу Эйлера факторрасслоения $Q / q$. Для вычисления класса Эйлера расслоения $Q / q$ введем комплексную структуру на расслоениях $T_{*} M, T^{*} M$ и $Q$. Для расслоения $T_{*} M$ определим умножение в слое на $i$ как поворот касательной плоскости на угол $\pi / 2$ (соответственно метрике и ориентации на поверхности). Это индуцирует комплексную структуру на расслоениях $T^{*} M$ и $Q$. При этом $q$ становится линейным комплексным подрасслоением расслоения $Q$. Легко показать, что для введенной комплексной 
структуры $q \simeq T^{*} M, Q \simeq T^{*} M \oplus T^{*} M^{\otimes_{\mathbb{C}}}$. Кроме того, $Q / q \simeq\left(T^{*} M\right)^{\otimes_{\mathbb{C}} 3}$. Следовательно, первый класс Чженя расслоения $Q / q$ равен $6 g-6=-3 \chi(M)$ [5], он же равен классу Эйлера расслоения $Q / q$. Теорема доказана.

\section{§4. Кубическая поверхность}

Напомним, что мы рассматриваем только вещественные кубические поверхности общего положения, комплексификации которых содержат ровно 27 различных проективных прямых, пересекающихся лишь попарно.

Для доказательства теоремы 4 нам потребуется несколько лемм. Следующие две леммы хорошо известны.

Лемма 5. Рассмотрим особую неприводимую вещественную кубическую кривую, имеющую двойную точку. Обе ветви кривой, проходящие через двойную точку, выпукль в малой окрестности этой двойной точки.

ЛЕмма 6. Плоскость, касающаяся двумерной поверхности в специальной гиперболической точке, локально пересекает эту поверхность по двум кривым, и специальная точка является точкой перегиба для обеих кривых.

СлЕдСТВИЕ ЛЕмм 5 и 6. (1) Каждая специальная гиперболическая точка кубической поверхности является пересечением некоторых двух прямых, лежащих на этой поверхности. Таким образом, кубическая поверхность, диффеоморфная $\mathbb{R P}^{2}$, содержит ровно 3 специальные гиперболические точки.

(2) Проективная плоскость, касающаяся кубической поверхности в специальной эллиптической точке, пересекает эту поверхность по одной проективной прямой.

ЛЕмма 7. Рассмотрим в трехмерном проективном пространстве $\mathbb{R P}^{3} \kappa у$ к бическую поверхность $\pi$, диффеоморфную двумерной проективной плоскости. Через каждую из трех проективных прямых этой поверхности можно провести ровно две плоскости, касающиеся поверхности в некоторой специальной эллиптической точке.

ДоКАЗАТЕЛЬСтво. Обозначим проективные прямые, лежащие на поверхности $\pi$, через $l_{1}, l_{2}, l_{3}$. Множество плоскостей, содержащих прямую $l_{1}$, является проективной прямой. Обозначим последнюю прямую через $l_{1}^{\prime}$. Сопоставим каждой точке поверхности $\pi-l_{1}$ плоскость, проходящую через эту точку и прямую $l_{1}$. Это задаст отображение из $\pi-l_{1}$ в прямую $l_{1}^{\prime}$, на прямой $l_{1}$ отображение доопределяется по непрерывности. Построенное отображение из $\pi$ в $l_{1}^{\prime}$ является стягиваемым ( $C^{\infty}$-гладким); поэтому разность числа локальных экстремумов и числа седловых критических точек этого отображения равна $\chi\left(\mathbb{R P}^{2}\right)=1$. Отображение имеет ровно одну седловую критическую точку - пересечение прямых $l_{2}$ и $l_{3}$; поэтому отображение обязано иметь ровно два локальных экстремума. Эти экстремумы как раз и являются специальными эллиптическими точками.

СледствиЕ. Кубическая поверхность, диффеоморфная $\mathbb{R P}^{2}$, содержит ровно 6 специильных эллиптических точек.

ЛЕмма 8. Рассмотрим в трехмерном проективном пространстве $\mathbb{R P}^{3} \kappa у$ бическую поверхность, диффеоморфную двумерной проективной плоскости. Пусть $l$ - некоторая проективная прямая на этой поверхности и $\gamma$ некоторая параболическая линия. Через прямую l можно провести касательную плоскость к некоторой специальной эллиптической точке, лежащей внутри $\gamma$, в том и только в том случае, когда $\gamma$ не касается $l$. 
ДокАЗАТЕЛЬСтво. Пусть Параболическая кривая $\gamma$ и прямая $l$ касаются в точке $P$. Обозначим через $\varphi$ отображение проекции кубической поверхности на прямую $l^{\prime}$, построенное в лемме 7. Покажем, что внутренность кривой $\gamma$ не содержит критических точек этого отображения. Пусть $H-$ специальная гиперболическая точка, являющаяся критической точкой отображения $\varphi$. Тогда значение $\varphi$ на внутренности кривой $\gamma$ заключено между $\varphi(H)$ и $\varphi(P)$. Действительно, кривые $\varphi^{-1}(\varphi(P))$ и $\gamma$ лежат по разные стороны от прямой $l$ в окрестности точки $P$ (это следует из простого вычисления); поэтому внутренности кривых не пересекаются (см. рис. 5). Таким образом, не существует проективной плоскости, содержащей прямую $l$ и касающейся внутренности кривой $\gamma$. Обратное утверждение леммы доказывается аналогично.

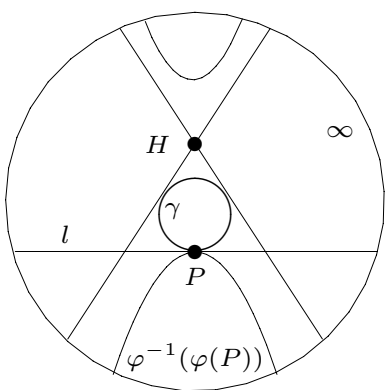

Рис. 5

ДоКАЗАТЕЛЬСТВо ТЕОРЕмЫ 4. Из леммы 8 вытекает последнее недоказанное утверждение теоремы - для любой параболической кривой на поверхности $\pi$ сумма числа специальных точек на этой кривой и числа специальных эллиптических точек внутри кривой равна трем. Теорема доказана.

Следующая лемма элементарна.

Лемма 9. Пусть кубическая поверхность состоит из двух компонент связности. Тогда одна из компонент обязана быть диффеоморфна сфере, а другая - проективной плоскости. Компонента, диффеоморфная сфере, выпукла.

ДоКАЗАТЕЛЬСТВО тЕОРЕмЫ 5. Рассмотрим кубическую поверхность $\pi$, состоящую из двух компонент связности. Компонента, диффеоморфная проективной плоскости, содержит ровно три проективные прямые. Через любую из этих трех прямых можно провести ровно две плоскости, касающиеся второй компоненты связности поверхности (так как эта компонента является выпуклой сферой, лемма 9). Отсюда (используя следствие лемм 5 и 6, п. (2)) получаем, что на сфере имеется ровно шесть специальных эллиптических точек. Теорема доказана.

\section{Гипотезы}

В заключение приведем две гипотезы.

ГиПотезА 1. Рассмотрим поверхность, лежащую в $\mathbb{R P}^{3}$ и диффеоморфную $\mathbb{R P}^{2}$. Эта поверхность содержит не менее 6 специальньх параболических точек, не менее 6 специиальньх эллиптических точек и не менее трех специиальных гиперболических точек, если точки учитываются с кратностями.

ЗАмЕчАНИЕ 1. Существует поверхность, являющаяся шевелением стандартного пространства $\mathbb{R} \mathbb{P}^{2}$, вложенного в $\mathbb{R} \mathbb{P}^{3}$, и содержащая только 4 геометрически 
различные специальные эллиптические точки. При этом одна из четырех точек имеет индекс 3 . Специальная эллиптическая точка поверхности $z=\left(x^{2}+y^{2}\right)+$ $\left(x^{2}+y^{2}\right)^{2}$ имеет индекс 3 .

ЗАмЕЧАНИЕ 2. Из результатов работы [6] вытекает справедливость гипотезы 1 для малых возмущений кубической поверхности $z=1 /\left(1+x^{2}+y^{2}\right)$.

Гипотеза 1 обобщает гипотезу Айкарди [4].

ГИПОТЕЗА 2. Поверхность в трехмерном пространстве, являющаяся графиком двоякопериодической функции, содержит в каждом периоде не менее четырех спецуиальных параболических точек, шести специиальных эллиптических точек и двух специиальных гиперболических точек.

\section{ЛИТЕРАТУРА}

1. Vassiliev V. A. Lagrange and Legendre characteristic classes. 2nd ed., Gordon \& Breach Publ., 1993. [Русский перевод: Васильев B. A. Лагранжевы и лежандровы характеристические классы. МЦНМО, М., 2000].

2. Платонова O. A. Проектирование гладких поверхностей. Труды семинара им. И. Г. Петровского, 10, 135-149 (1984).

3. Segre B. The nonsingular cubic surfaces. Clarendon Press, Oxford, 1942.

4. Арнольд В. И. Топологические вопросы теории распространения волн. УМН, 51, вып. 1, 3-50 (1996).

5. Ботm Р., Ту Л. В. Дифференциальные формы в алгебраической топологии. Наука, M., 1989.

6. Арнольд В. И. Замечания о параболических кривых на поверхностях и многомерной теории Мёбиуса-Штурма. Функц. анализ и его прил., 31, вып. 4, 3-18 (1997).

Независимый московский университет

Поступило в редакцию 22 декабря 1998 г. 